\title{
Dynamic range of hypercubic stochastic excitable media
}

\author{
Vladimir R. V. Assis:* and Mauro Copelli \\ Laboratório de Física Teórica e Computacional, Departamento de Física, \\ Universidade Federal de Pernambuco, 50670-901 Recife, PE, Brazil
}

\begin{abstract}
We study the response properties of $d$-dimensional hypercubic excitable networks to a stochastic stimulus. Each site, modelled either by a three-state stochastic susceptible-infected-recoveredsusceptible system or by the probabilistic Greenberg-Hastings cellular automaton, is continuously and independently stimulated by an external Poisson rate $h$. The response function (mean density of active sites $\rho$ versus $h$ ) is obtained via simulations (for $d=1,2,3,4$ ) and mean-field approximations at the single-site and pair levels $(\forall d)$. In any dimension, the dynamic range and sensitivity of the response function are maximized precisely at the nonequilibrium phase transition to self-sustained activity, in agreement with a reasoning recently proposed. Moreover, the maximum dynamic range attained at a given dimension $d$ is a decreasing function of $d$.

PACS numbers: 87.19.L-, 87.10.-e, 87.18.Sn, 05.45.-a
\end{abstract}

\section{INTRODUCTION}

The building blocks of sensory organs are excitable neurons, upon which physical stimuli impinge continuously. Information about these stimuli is transformed into electrical activity of the neuronal membranes, usually in the form of nonlinear excitations called spikes. The biophysics of ion channels involved in this process has been thoroughly investigated in the last five decades, after the success of the Hodgkin-Huxley theory [1]. Despite immense progress in this regard, however, some fundamental questions have remained unanswered. One of them has to do with two apparently conflicting experimental results. On the one hand, animals are subjected to stimulus intensities spanning many orders of magnitude, which their brains somehow manage to handle. This result is perhaps most easily revealed in psychophysical experiments. When humans are asked to assign an arbitrary (psychological) value to a given physical stimulus, this value is shown to be proportional to a power $m$ of the stimulus intensity [2] (Stevens' law of psychophysics). The fact that the Stevens' exponent $m$ is usually $<1$ is consistent with the large dynamic range and sensitivity of psychophysical response functions (for instance, $m \simeq 0.6$ for the smell of heptane [2]). On the other hand, the response (mean firing rate) of isolated sensory neurons as a function of stimulus intensity has been shown to be an approximately linear saturating curve, at least for some sensory modalities. This implies that their dynamic range is usually small (for olfactory sensory neurons, they stay in the range of $\sim 10 \mathrm{~dB}[\underline{3}, 4]$ ).

How is it then that large dynamic ranges are obtained from elements which individually have small dynamic ranges? What is the mechanism that generates Stevens' exponents $m<1$ ? Two main mechanisms have been historically recognized as contributing to the phenomenon:

\footnotetext{
*Electronic address: vladimirassis@df.ufpe.br

${ }^{\dagger}$ Electronic address: mcopelli@df.ufpe.br corresponding author
}

one of them invokes the intrinsic variation of thresholds in a population of sensory neurons [5]. The second one is adaptation, by which neurons adjust their operating ranges according to the statistics of the ambient stimulus (see, e.g., Refs. [6, 7, 8, 9, 10] for the case of the visual system). Both mechanisms certainly contribute to an enhancement of dynamic range. However, recent experimental data strongly suggest that additional mechanisms based on a collective neuronal phenomenon could be at play: Deans et al. 11] showed that knocking out gap junctions (electrical synapses among neurons) leads to a substantial change in the response function of retinal ganglion cells of mammals, with a decrease in dynamic range and sensitivity [11] and an increase in the response exponent [12].

In the last few years we have investigated this third possible mechanism, which addresses how neurons could cooperatively lead to an enhancement of dynamic range owing to the presence of lateral interactions (e.g. via chemical or electrical synapses). Connected, they form an extended system in which excitable waves are created upon incidence of incoming stimuli and annihilated upon collision (either with one another or with boundaries) due to the nonlinearity of their dynamics. The overall effect of this process is to collectively produce an enhancement of dynamic range and sensitivity, as compared to those of the elements alone [12, 13, 14, 15, 16, 17, 18]. We emphasize that our proposal relies on very basic properties of excitable media, which opens the possibility that it could be applied not only to sensory systems, but wherever else enhanced sensitivity and dynamic range are required. For example, extreme sensitivity is observed in rat motor cortex, where stimulation of a single pyramidal cell can evoke whisker movements [19]. Also potentially related to what we propose is the experimental observation that electrical synapses in the neocortex are present exclusively between inhibitory interneurons [20]. Electrical coupling allows them to excite each other [20] and might have the functional role of augmenting their dynamic range and sensitivity.

The mechanism we discuss here is simple: let the ar- 
rival of a suprathreshold stimulus reaching a sensory neuron be modelled by a Poisson process with rate $h$, which would be proportional to the stimulus intensity (say, the concentration of an odorant reaching the olfactory epithelium). For very small $h$, stimulus events are rare and each of them would produce on average one excitation, if the excitable elements were disconnected. If they are connected, however, excitations can propagate stochastically to neighbors at some rate $\lambda$. In this case, a single-stimulus event will generate an amplifying excitable wave. If $\lambda$ is small, this wave will die out after some time, but the average network activity $\rho$ will nonetheless be larger than that of uncoupled neurons, leading to an enhanced sensitivity and larger dynamic range. This amplifying effect becomes more pronounced as $\lambda$ increases, so the dynamic range initially increases with $\lambda$. Increasing $\lambda$ further, however, one may reach a phase transition at some critical value $\lambda=\lambda_{c}$, above which self-sustained activity becomes stable $\left[\rho\left(h=0 ; \lambda>\lambda_{c}\right)>0\right]$. In this supercritical regime, the larger the coupling $\lambda$, the more difficult it becomes for $\rho$ to code for weak stimuli, which can hardly be distinguished from the self-sustained background activity of the network. Therefore, for $\lambda>\lambda_{c}$ the dynamic range decreases with increasing $\lambda$. Putting those two results together, one concludes that the dynamic range is maximum at criticality [16].

Clearly, the above reasoning applies to essentially any network topology. In its original version [16], it was formulated for an Erdős-Rényi random graph, where a simple mean-field model perfectly captured the phenomenon. However, the enhancement of the dynamic range in that topology was about $50 \%$, which is much less than what is observed experimentally (for instance, dynamic ranges in the olfactory glomerulus are at least twice as large as in olfactory sensory neurons [21, 22]). This raises the question whether networks with different topologies could yield larger dynamic ranges and, if so, how these depend on network structural parameters. In Ref. 18], for instance, the dynamic range of scale-free networks was shown to depend on the density of loops, but the evidence relied only on numerical simulations. In this contribution, we make use of simulations and analytical methods to deal with this question in hypercubic lattices, looking at the dimension $d$ as a parameter.

\section{MODEL AND SIMULATION RESULTS}

We explore these ideas within a simple stochastic model of pulse-coupled excitable elements:

$$
\begin{aligned}
\dot{P}_{t}\left(S_{x}\right) & =-h P_{t}\left(S_{x}\right)-\lambda \sum_{y} P_{t}\left(S_{x}, I_{y}\right)+\gamma P_{t}\left(R_{x}\right)(1) \\
\dot{P}_{t}\left(I_{x}\right) & =-P_{t}\left(I_{x}\right)+\lambda \sum_{y} P_{t}\left(S_{x}, I_{y}\right)+h P_{t}\left(S_{x}\right) \\
\dot{P}_{t}\left(R_{x}\right) & =-\gamma P_{t}\left(R_{x}\right)+P_{t}\left(I_{x}\right)
\end{aligned}
$$

where $P_{t}\left(\alpha_{x}\right)$ is the probability that site at location $x \in\{1, \ldots, N\}$ is in state $\alpha$ at time $t ; P_{t}\left(\alpha_{x}, \beta_{y}\right)$ is the joint probability that sites at locations $x$ and $y$ are respectively in states $\alpha$ and $\beta$ at time $t ; \alpha, \beta \in\{S, I, R\}$ denote a quiescent, excited or refractory state, respectively; $y$ runs over the neighborhood of $x$; and $\gamma^{-1}$ is the characteristic refractory time, measured in units of the characteristic excitation time (defined as 1, without loss of generality [23]). We employ the notation of the stochastic susceptible-infected-recovered-susceptible (SIRS) model, to which this model is identical except for the externalstimulus field $h$, which is often missing in epidemiological modelling (where it amounts to a spontaneous infection rate 24]). We can therefore extend a previous analysis of the stochastic SIRS model on a hypercubic lattice by Joo and Lebowitz [23], from which our results can be derived if the external field $h$ is added as in Eqs. (11)-(3).

We are interested in the response function (or transfer function) of the excitable medium - i.e. the dependence of the stationary density of active sites $\rho \equiv \lim _{t \rightarrow \infty} P_{t}(I)$ on the external stimulus intensity $h$ (note that, in the context of neuroscience, the mean firing rate can be obtained by dividing $\rho$ by the mean excitation time). Figure 1shows simulation results which confirm the general scenario described above. For $\gamma=1$ (which is kept fixed throughout this paper) and $d=2$, a phase transition occurs at $\lambda=\lambda_{c} \simeq 0.567(2)$. In the subcritical regime $\left(\lambda<\lambda_{c}\right) \rho(h)$ is a linear-saturating curve whose slope increases monotonically with $\lambda$. In the supercritical regime $\left(\lambda>\lambda_{c}\right)$ one has $\rho_{0} \equiv \lim _{h \rightarrow 0} \rho(h)>0$, with $\rho_{0}$ increasing monotonically with $\lambda$ [23].

To obtain the dynamic range of the response curve, we employ the definition usually adopted in the biological literature [3, 4]. Let $h_{0.1}$ and $h_{0.9}$ be the stimulus intensities such that their corresponding responses $\left(\rho_{0.1}\right.$ and $\left.\rho_{0.9}\right)$ are, respectively, $10 \%$ and $90 \%$ above the base-line activity within the range $\left[\rho_{0}, \rho_{\max }\right]$ :

$$
\rho_{\eta} \equiv \rho_{0}+\eta\left(\rho_{\max }-\rho_{0}\right),
$$

where $\rho\left(h_{\eta}\right) \equiv \rho_{\eta}, \rho_{\max } \equiv \lim _{h \rightarrow \infty} \rho(h)=\gamma /(\gamma+1)$, and $\eta \in\{0.1,0.9\}$. The dynamic range $\Delta$ is defined as

$$
\Delta \equiv 10 \log _{10}\left(\frac{h_{0.9}}{h_{0.1}}\right) .
$$

As depicted in Fig. 1(a), $\Delta$ amounts to the range of stimulus intensities (measured in $\mathrm{dB}$ ) that can be appropriately coded by the average activity in the network, discarding stimuli whose responses are too close either to baseline activity $\left(\rho<\rho_{0.1}\right)$ or to saturation $\left(\rho>\rho_{0.9}\right)$.

According to this standard definition, the dynamic range of the response curves in Fig. 1(a) shows the predicted behavior: for $\lambda<\lambda_{c}\left(\lambda>\lambda_{c}\right), \Delta(\lambda)$ is a monotonically increasing (decreasing) function. As shown in Fig. 2, the maximum dynamic range occurs precisely at criticality, where the response function is governed by the scaling relation $\rho \sim h^{\delta_{h}^{-1}}$. In all our simulations, the 

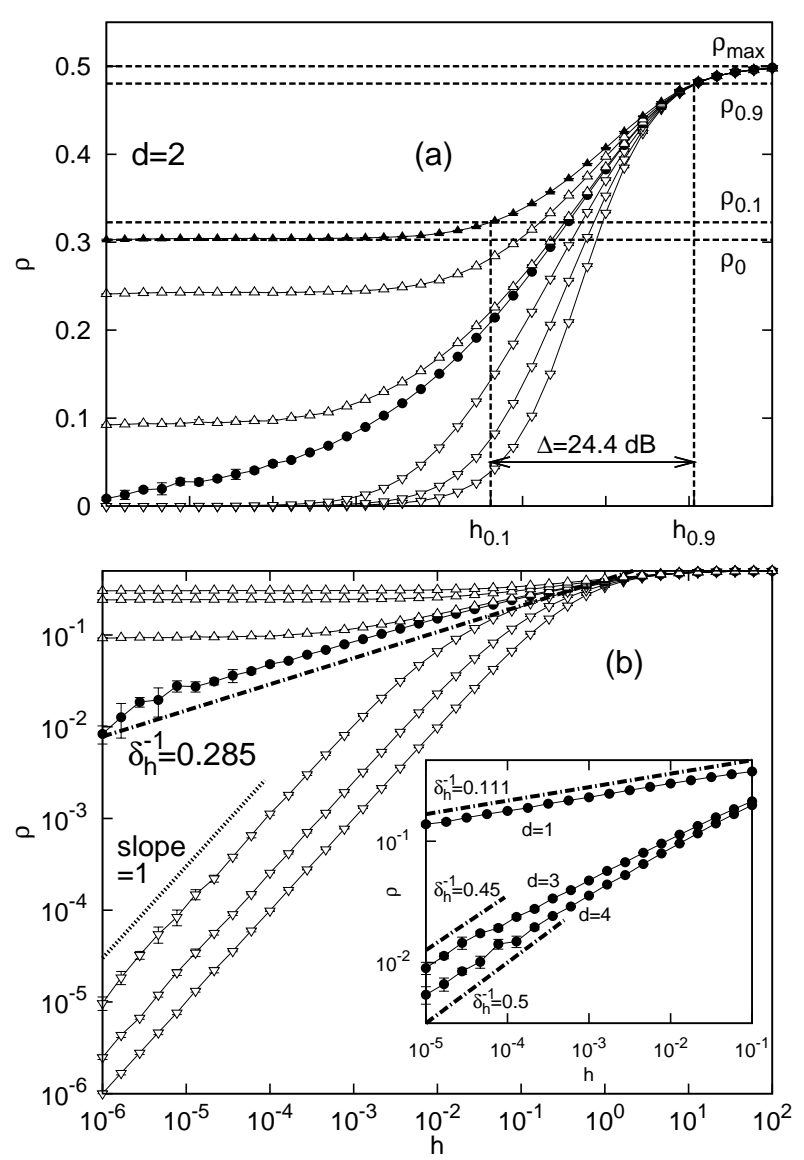

FIG. 1: Response curves $\rho(h)$ of the stochastic SIRS model on a two-dimensional lattice (results from simulations with $N=100^{2}$ sites, periodic boundary conditions, averaged over a maximum time $T_{\max } \sim 10^{4}-10^{6}$ and five runs) in (a) linear-log scale and (b) log-log scale. From bottom to top, triangles denote $\lambda=0,0.2, \ldots, 1$. Filled circles denote $\lambda \simeq$ $\lambda_{c}$. Relevant parameters for calculating the dynamic range $\Delta$ are exemplified in (a) for $\lambda=1$ (filled triangles). (b) Dotdashed lines show literature values for the critical exponent $\delta_{h}^{-1}$. Inset: response function near criticality for $d=1,3$ and 4 (system sizes and approximate critical coupling are $N=5000$ and $\lambda_{c} \simeq 7.73(8), N=20^{3}$ and $\lambda_{c} \simeq 0.259(3), N=10^{4}$ and $\lambda_{c} \simeq 0.167(2)$, respectively).

observed critical exponent $\delta_{h}^{-1}$ is compatible with the literature values for the directed percolation (DP) universality class: namely, $\delta_{h}^{-1}=0.111,0.285,0.45$, and $1 / 2$ for $d=1,2,3$, and $d \geq 4$, respectively [25, 26, 27] [see inset of Fig. 1(b)]. This should be expected, since the model has local rules, a continuous transition to a unique absorbing state, and no further conservation laws [24, 28, 29, 30]. Because the exponent $\delta_{h}^{-1}$ increases with increasing $d$ and since it is the key element governing the dynamic range at criticality, we come to one of the main results of this paper: the maximum dynamic range attained at a given dimension $d$ is a decreasing function of $d$. This result is summarized in Fig. 3, which exhibits the peaks of Fig. 2 versus the dimension of the lattice.
We further note that similar results are obtained if one employs the Greenberg-Hastings cellular automaton (GHCA), where now the state transition $S \rightarrow I$ is controlled by probabilities $p_{h}$ (external stimulus) and $p_{\lambda}$ (coupling), $I \rightarrow R$ by $p_{\delta}$ and $R \rightarrow S$ by $p_{\gamma}$ [31]. The only difference occurs for the particular case of excitations with a deterministic duration $\left(p_{\delta}=1\right)$ in $d=1$, in which case self-sustained activity cannot be stable and the maximum dynamic range occurs for $p_{\lambda}=1$ with an anomalous response exponent $1 / 2$, as previously reported [12] (see stars in Fig. 3). Note that $p_{\delta}=1$ seems biologically more realistic for neurons: while coupling may be stochastic, the duration of a spike is generally well described by a deterministic dynamics. For $p_{\delta}<1$ and $d=1$ a phase transition can occur just like in the SIRS model, and in this case $\Delta\left(\lambda_{c}\right)$ is again a monotonically decreasing function of $d$ (as exemplified for $p_{\delta}=0.5$; see squares in Fig. 33).

\section{MEAN-FIELD RESULTS}

\section{A. Single-site approximation}

The remainder of this paper focuses on the possibilities of understanding these results with analytical means. Clearly, solving Eqs. (11)-(3) for $\lambda \neq 0$ is difficult, because the dynamics of single-site probabilities $P_{t}\left(\alpha_{x}\right)$ depend on two-site joint probabilities $P_{t}\left(\alpha_{x}, \beta_{y}\right)$, which in their turn depend on higher-order terms, and so forth. The simplest way to truncate this infinite hierarchy of equations is the single-site (1S) mean field approximation, in which correlations are neglected [23, 24]. The conditional probabilities are approximated as $P_{t}\left(\alpha_{x} \mid \beta_{y}\right) \stackrel{1 S}{\simeq} P_{t}\left(\alpha_{x}\right)$, which implies that $m$-site joint probabilities get factorized as $P\left(\alpha_{x_{1}}^{(1)}, \ldots, \alpha_{x_{m}}^{(m)}\right) \stackrel{1 S}{\simeq} \prod_{j=1}^{m} P_{t}\left(\alpha_{x_{j}}^{(j)}\right)$, where $\alpha^{(j)} \in$ $\{S, I, R\}$. In this approximation, assuming homogeneity and isotropy, Eqs. (1)-(3) reduce to a closed system and one obtains

$$
\begin{aligned}
\rho(h) \stackrel{1 S}{=} & \left(\frac{\rho_{\max }}{2 \sigma}\right)\left(\rho_{\max }^{-1} h+1-\sigma\right) \times \\
& \left\{-1+\sqrt{1+\frac{4 \sigma \rho_{\max }^{-1} h}{\left(\rho_{\max }^{-1} h+1-\sigma\right)^{2}}}\right\} .
\end{aligned}
$$

Note that information about the dimension $d$ of the network is absorbed into an effective branching parameter $\sigma \equiv \lambda z$, where $z \equiv 2 d$ is the number of neighbors each site has. When $\sigma \rightarrow 0$, we obtain a linear saturating response $\rho(h)=\rho_{\max } h /\left(\rho_{\max }+h\right)$, which is an exact result for uncoupled excitable elements. If $h \rightarrow 0$, Joo and Lebowitz's 1S results are recovered: without an external stimulus, the $1 \mathrm{~S}$ approximation predicts a phase transition at $\sigma=\sigma_{c}=1\left(\lambda=\lambda_{c}=1 / z\right)$, above which an active phase with $\rho_{0}=\rho_{\max } \sigma(\sigma-1)>0$ is stable [23]. The weak stimulus response is linear in the subcritical regime, $\rho\left(h ; \lambda<\lambda_{c}\right) \stackrel{1 S}{\simeq} h /(1-z \lambda)$. At criticality, how- 

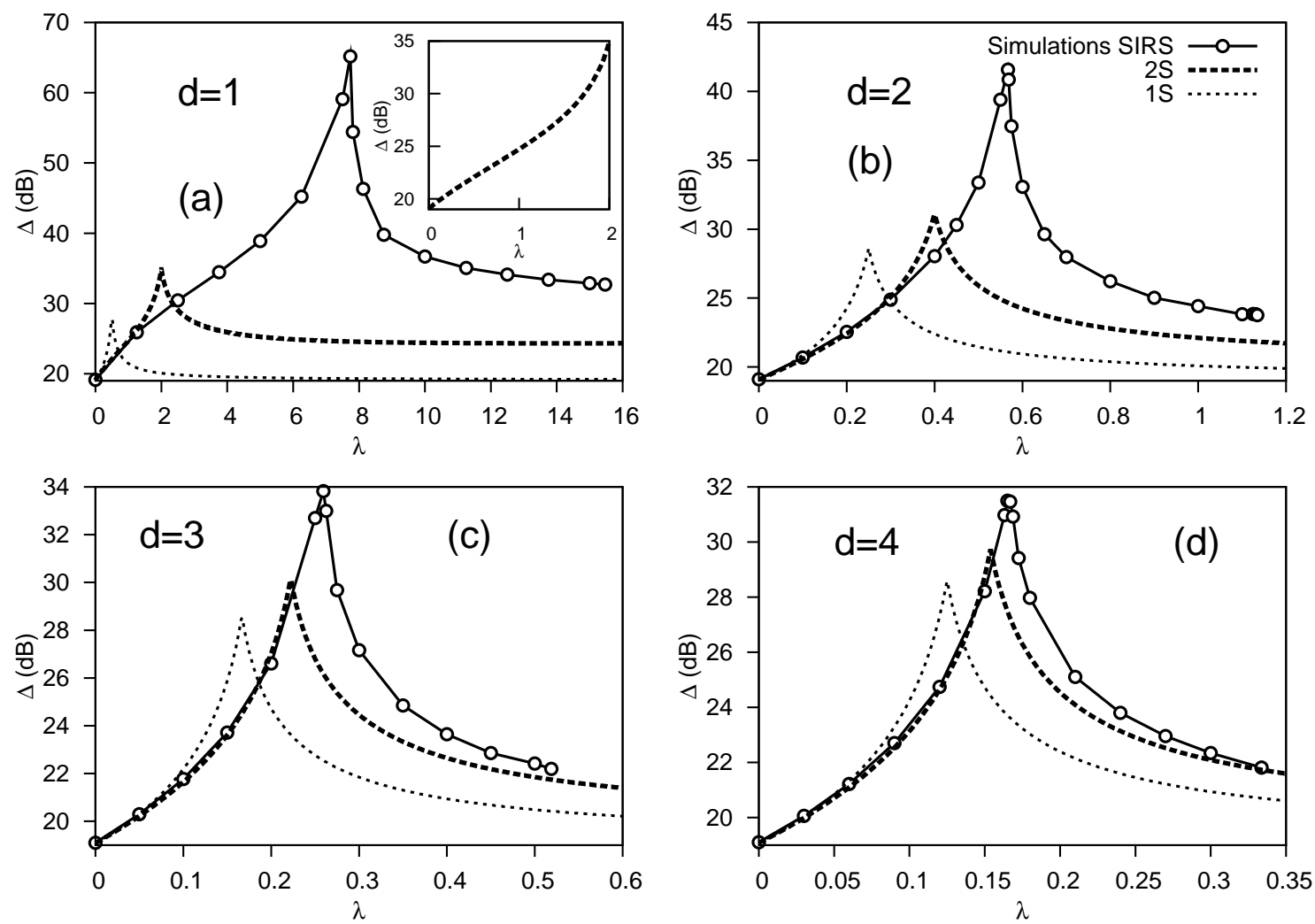

FIG. 2: Dynamic range vs. coupling: simulation results (symbols), 1S (thin dashed) and 2S (thick dashed) mean field approximations. The peaks occur always at the phase transition. Half-widths of tuning of the critical regime [with heights measured relative to $\Delta(\lambda=0)]$ are $\Delta \lambda \simeq 1.4,0.094,0.059$ and 0.042 for $d=1,2,3$ and 4 , respectively.

ever, it is governed by $\rho\left(h ; \lambda=\lambda_{c}\right) \stackrel{1 S}{\simeq}\left(\rho_{\max } h\right)^{1 / 2}$, which leads to the mean-field exponent $\delta_{h}^{-1}=1 / 2$. Applying the definition of Eqs. (4) and (5) to Eq. (6), one obtains the dynamic range in the $1 \mathrm{~S}$ approximation:

$$
\Delta(\sigma) \stackrel{1 S}{=} \begin{cases}10 \log _{10}\left[81\left(\frac{1-0.1 \sigma}{1-0.9 \sigma}\right)\right] & (\sigma \leq 1) \\ 10 \log _{10}\left[81\left(\frac{\sigma-0.1}{\sigma-0.9}\right)\right] & (\sigma \geq 1) .\end{cases}
$$

As depicted in Fig. 2, the peak at $\sigma=1$ is $\Delta\left(\sigma_{c}\right)=$ $30 \log _{10} 9 \mathrm{~dB}$, which corresponds to an exact $50 \%$ enhancement as compared to uncoupled elements: $\Delta(0)=$ $20 \log _{10} 9 \mathrm{~dB}$. Not surprisingly, this result holds for all $d$.

\section{B. Pair approximation}

To gain analytical insight into the dependence of $\Delta$ on $d$, we have solved Eqs. (1)-(3) in the so-called pair or two-site (2S) approximation, in which conditional probabilities are truncated beyond nearest neighbors: $P\left(\alpha_{x_{1}} \mid \beta_{x_{2}}, \chi_{x_{3}}\right) \stackrel{2 S}{\simeq} P\left(\alpha_{x_{1}} \mid \beta_{x_{2}}\right)$ (this approximation is valid for the hypercubic lattice because $x_{3}$ is not a nearest neighbor of $x_{1}$ ). This leads to three-site joint probabilities being approximated as $P\left(\alpha_{x_{1}}, \beta_{x_{2}}, \chi_{x_{3}}\right) \stackrel{2 S}{\simeq}$ $P\left(\alpha_{x_{1}}, \beta_{x_{2}}\right) P\left(\beta_{x_{2}}, \chi_{x_{3}}\right) / P\left(\beta_{x_{2}}\right)$. To arrive at a closed set of equations, first one has to write down the dynamics also for two-site probabilities, which in this case are a direct extension of Joo and Lebowitz's equations for $h \neq 0$ [23]. These equations (A.1)-(A.3) can be found in the Appendix. Applying the $2 \mathrm{~S}$ approximation to all equations, one concludes, after some manipulation, that $\rho$ satisfies a cubic equation [Eq. (A.9)]. For $h=0$ one can obtain the $2 \mathrm{~S}$ value of the coupling at which the phase transition occurs: $\lambda_{c} \stackrel{2 S}{=}(\gamma+1) /(2 d-2+(2 d-1) \gamma)[23]$.

Figure 2] shows the dynamic ranges calculated from the numerically obtained response curves. The $2 \mathrm{~S}$ approximation shows a better agreement with simulations than does the $1 \mathrm{~S}$ approximation, and Fig. 2 clearly shows how the agreement improves with increasing $d$. In particular, note that the $2 \mathrm{~S}$ approximation reproduces the inflection in the $\Delta(\lambda)$ curve, which appears only for $d=1$ [inset of Fig. 2(a)]. Like in the $1 \mathrm{~S}$ mean-field approximation and in the simulations, the subcritical response function for weak stimuli is linear:

$$
\rho\left(h ; \lambda<\lambda_{c}\right) \stackrel{2 S}{\simeq}\left\{\frac{\gamma+1+\lambda(\gamma+2)}{[2 d-2+(2 d-1) \gamma]\left(\lambda_{c}-\lambda\right)}\right\} h .
$$

More important for the topic of this article, the $2 \mathrm{~S}$ approximation manages to capture the dependence of $\Delta\left(\lambda_{c}\right)$ on $d$, despite the fact that the weak-stimulus response at the critical coupling is still governed by a mean-field ex- 


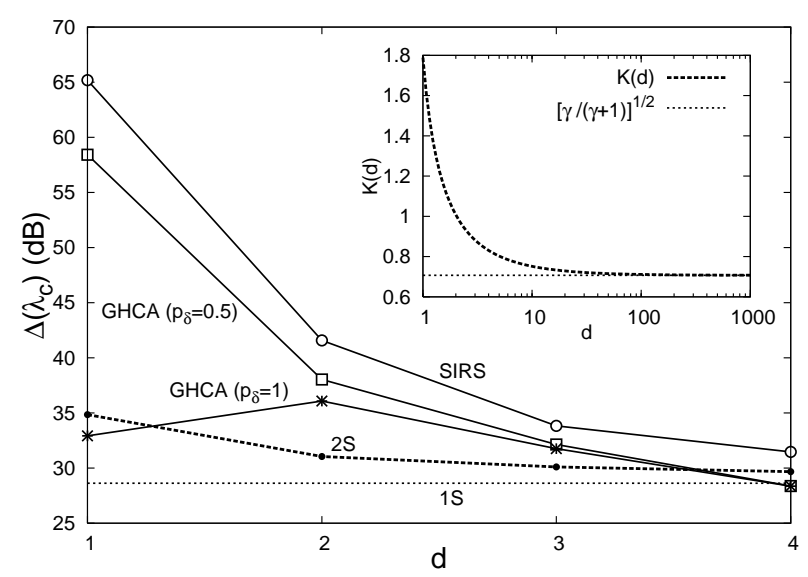

FIG. 3: Maximum dynamic range vs. dimension of the lattice: simulation results for the SIRS (open circles) and GHCA (stars denote $p_{\delta}=1$ and squares denote $p_{\delta}=0.5$ ) models, $1 \mathrm{~S}$ (thin dashed) and 2S (thick dashed) approximations. GHCA simulations were performed with $p_{\gamma}=1 / 3$ and the same number of sites, $T_{\max }$ and number of runs as described in the caption of Fig. 1 for the SIRS model. Inset: $K(d)$ approaches the $1 \mathrm{~S}$ coefficient $\rho_{\max }^{1 / 2}$ in the limit $d \rightarrow \infty$ (see eq. 9).

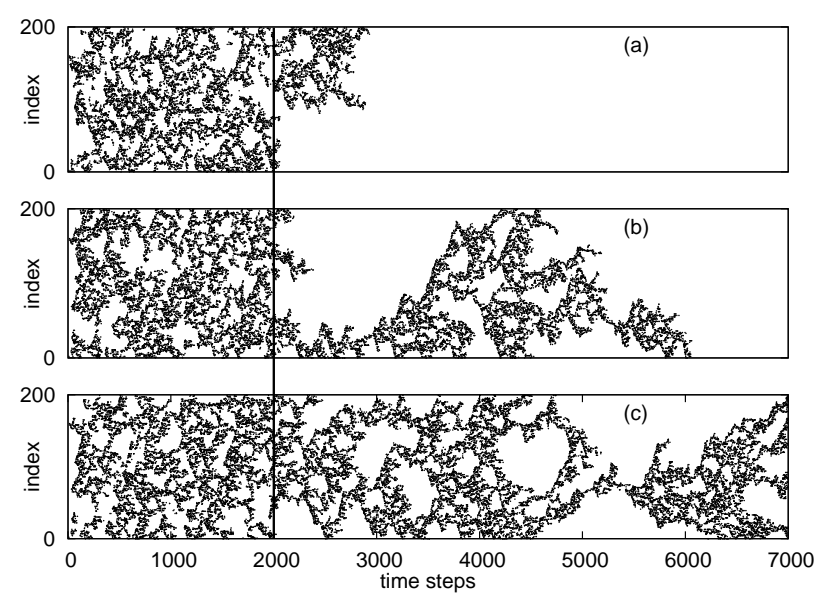

FIG. 4: Active sites vs. time in a single run of a small $d=1$ SIRS system (one time step is counted every $N=200$ updates; boundary conditions are open). An external stimulus with rate $h=10^{-2}$ is applied for $0<t<2000$ ( $h=0$ otherwise). Upper, middle and lower panels show the subcritical $(\lambda=7.0)$, critical $(\lambda=7.73)$ and supercritical $(\lambda=8.5)$ regimes, respectively.

ponent: $\rho\left(\lambda_{c} ; d\right) \stackrel{2 S}{\simeq} K(d) h^{1 / 2}$. In this case, the decreasing function

$$
K(d)=\sqrt{\frac{4 d^{2} \gamma(\gamma+1)^{2}(2 d-1)^{-1}}{\gamma^{3}(2 d-1)+\gamma^{2}(6 d-4)+\gamma(6 d-5)+2 d-1}}
$$

correctly incorporates the influence of $d$ on $\Delta\left(\lambda_{c}\right)$ (see inset of Fig. 31).

The fact that neither the $1 \mathrm{~S}$ nor $2 \mathrm{~S}$ approximations can reproduce the correct critical exponents for $d<4$ is well known. Inherent to those mean-field approximations is the truncation of correlations, which is clearly inconsistent with the observed divergence of the correlation length $\xi$ and relaxation time $\tau$ as criticality is approached: $\xi \sim\left|\lambda-\lambda_{c}\right|^{-\nu_{\perp}}$ and $\tau \sim\left|\lambda-\lambda_{c}\right|^{-\nu_{\|}}$, where $\nu_{\perp}>0$ and $\nu_{\|}>0$ are critical exponents [24]. In our simulations, correlation lengths and relaxation times at criticality are limited only by system size. At criticality also the survival probability $\mathcal{P}(t)$ decays as a power law (as opposed to an exponential fall in the subcritical regime) 24], which can lead to long-lived excitation

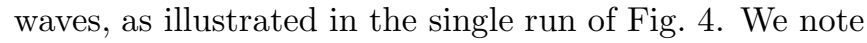
that correlations among cortical neurons several synapses distant from one another have been experimentally observed [32] and associated with the propagation of electrical waves [33].

\section{CONCLUDING REMARKS}

We have presented an analysis (with simulations and analytical results) of the response function of the stochastic SIRS model on hypercubic lattices. We confirmed that, as argued in Ref. [16], the maximum dynamic range is obtained precisely at the nonequilibrium phase transition where self-sustained activity becomes stable. Moreover, since the response function at criticality is governed by the critical exponent $\delta_{h}^{-1}$, which for the DP universality class increases with $d$, the maximum dynamic range obtained at a given dimension is a decreasing function of $d$. We therefore corroborate the claim that networks with spatial organization may have larger dynamic ranges than the random networks for which these ideas were first developed [16].

This suggests the usefulness of low-dimensional arrays of excitable units for artificial sensor design, as well as raises speculations regarding the effective dimensionality of living neural networks. If one admits that large dynamic ranges could be favored by natural selection, organisms would tend to have their brains tuned at criticality, and in this case the mystery of how Stevens' exponents $<1$ arise would be solved: they would just be the critical exponent $\delta_{h}^{-1}[16]$.

In this context, our theoretical results join a recent flow of experimental evidence which is compatible with neurons collectively operating in a critical regime 11,34 , 35, 36, 37, 38]. It is interesting to note that these experiments very often reveal exponents close to mean-field values: for instance, in cultures and acute slices of rat cortex, spontaneous activity occurs in avalanches whose size distribution decays as a power law with an exponent $3 / 2$ [34, 35, 36], which is the mean field result for branching processes. Also, the response function of retinal ganglion cells is well fitted by $\rho \sim h^{0.58}[11,12]$, yielding an exponent which is remarkably close to the Stevens' exponent for light intensity [2] and the DP $\delta_{h}^{-1}$ exponent for $d=4$. It would be interesting to investigate whether a small-world connectivity could conciliate these results, 
on the one hand preserving the local order observed in real neural networks (see [39] for a recent review) while on the other hand allowing for mean-field exponents owing to a small density of long-range connections.

\section{Acknowledgments}

V.R.V.A and M.C. acknowledge financial support from CAPES, Conselho Nacional de Desenvolvimento Científico e Tecnológico (CNPq), FACEPE, and special program PRONEX. The authors are grateful to R. Dickman, M. A. F. Gomes, J. Joo, S. C. Ferreira Jr., O. Kinouchi, D. R. Chialvo, and an anonymous referee for discussions and suggestions.

\section{APPENDIX: EQUATIONS FOR THE TWO-SITE APPROXIMATION}

As mentioned previously, the equations in Ref. [23] can be easily extended by including an external field $h$ as follows:

$$
\begin{aligned}
\dot{P}_{t}\left(S_{x}, I_{y}\right)= & \gamma P_{t}\left(R_{x}, I_{y}\right)-(\lambda+1+h) P_{t}\left(S_{x}, I_{y}\right) \\
& +h P_{t}\left(S_{x}, S_{y}\right)+\sum_{w \in \mathcal{N}^{x}(y)} \lambda P_{t}\left(S_{x}, S_{y}, I_{w}\right) \\
- & \sum_{w \in \mathcal{N}^{y}(x)} \lambda P_{t}\left(I_{w}, S_{x}, I_{y}\right) \\
\dot{P}_{t}\left(S_{x}, R_{y}\right)= & P_{t}\left(S_{x}, I_{y}\right)+\gamma P_{t}\left(R_{x}, R_{y}\right) \\
& -(\gamma+h) P_{t}\left(S_{x}, R_{y}\right) \\
& -\sum_{w \in \mathcal{N}^{y}(x)} \lambda P_{t}\left(I_{w}, S_{x}, R_{y}\right) \quad(\mathrm{A} .2) \\
\dot{P}_{t}\left(R_{x}, I_{y}\right)= & -(\gamma+1) P_{t}\left(R_{x}, I_{y}\right)+P_{t}\left(I_{x}, I_{y}\right) \\
& +h P_{t}\left(R_{x}, S_{y}\right) \\
& +\sum_{w \in \mathcal{N}^{x}(y)} \lambda P_{t}\left(R_{x}, S_{y}, I_{w}\right),
\end{aligned}
$$

where $\mathcal{N}^{x}(y)$ is the neighborhood of $y$, excluding $x$. Note that we can omit the equation for $P_{t}\left(I_{x}, I_{y}\right)$ because of the normalization condition $\sum_{A} P_{t}\left(A_{x}, B_{y}\right)=$ $P_{t}\left(B_{y}\right)$ [the same reasoning applies to $P_{t}\left(S_{x}, S_{y}\right)$ and $\left.P_{t}\left(R_{x}, R_{y}\right)\right]$. By applying the two-site approximation to Eqs. (1)-(3) and Eqs. (A.1)-(A.3) under homogeneity and isotropy assumptions [23], as well as the normalization condition $\sum_{A} P_{t}\left(A_{x}\right)=1$, we obtain

$$
\begin{aligned}
\dot{P}_{t}(S)= & \gamma-(\gamma+h) P_{t}(S)-\gamma P_{t}(I) \\
& -z \lambda P_{t}(S, I), \\
\dot{P}_{t}(I)= & h P_{t}(S)-P_{t}(I)+z \lambda P_{t}(S, I), \\
\dot{P}_{t}(S, I)= & h P_{t}(S)-(\lambda+1+2 h) P_{t}(S, I)-h P_{t}(S, R) \\
& +\gamma P_{t}(R, I) \\
& +(z-1) \lambda \frac{P_{t}(S, I)}{P_{t}(S)}\left[P_{t}(S)-2 P_{t}(S, I)\right. \\
& \left.-P_{t}(S, R)\right], \\
\dot{P}_{t}(S, R)= & \gamma-\gamma P_{t}(S)-\gamma P_{t}(I)+P_{t}(S, I) \\
& -(2 \gamma+h) P_{t}(S, R)-\gamma P_{t}(R, I) \\
& -(z-1) \lambda \frac{P_{t}(S, I) P_{t}(S, R)}{P_{t}(S)},
\end{aligned}
$$

$$
\begin{aligned}
\dot{P}_{t}(R, I)= & P_{t}(I)-P_{t}(S, I)+h P_{t}(S, R)-(2+\gamma) P_{t}(R, I) \\
& +(z-1) \lambda \frac{P_{t}(S, I) P_{t}(S, R)}{P_{t}(S)} .
\end{aligned}
$$

The above equations form a closed system of ordinary differential equations. In its fixed point, $\rho=$ $\lim _{t \rightarrow \infty} P_{t}(I)$ is shown to satisfy the cubic equation

$$
A_{1} \rho^{3}+A_{2} \rho^{2}+A_{3} \rho+A_{4}=0,
$$

where

$$
\begin{aligned}
A_{1}= & \gamma^{2}\left\{\gamma^{3}\left[z^{2}(z-1) \lambda-z\right]+\gamma^{2}\left[z\left(2 z^{2}-2 z-1\right) \lambda-2 z\right.\right. \\
& -1]+\gamma\left[2 z\left(z^{2}-z-1\right) \lambda-2 z-1\right]+z\left[\left(z^{2}-z-1\right) \lambda\right. \\
& -1]\}-h\left\{h^{2}(\gamma+1)^{4}+h\left[\gamma^{5} z+\gamma^{4}(z \lambda+4 z+3)+\right.\right. \\
& \gamma^{3}(4 z \lambda+7 z+9)+\gamma^{2}(6 z \lambda+7 z+9)+\gamma(4 z \lambda+ \\
& 4 z+3)+z \lambda+z]+\gamma\left\{\gamma^{4}\left(z^{2} \lambda+2 z\right)+\gamma^{3}[z \lambda(3 z+\right. \\
& 2)+6 z+3]+2 \gamma^{2}[z(2 z+3) \lambda+4 z+3]+3 \gamma[z(z+ \\
& 2) \lambda+2 z+1]+z[(z+2) \lambda+2)]\}\}, \quad \text { (A.10) } \\
& \\
A_{2}= & \gamma\left\{z \gamma ^ { 2 } \left\{\gamma^{2}[-2 z(z-1) \lambda+z+1]+\gamma\left[-\left(3 z^{2}-4 z\right.\right.\right.\right. \\
& \left.-1) \lambda+z+3]-\left(2 z^{2}-3 z-1\right) \lambda+z+1\right\}+ \\
& h\left\{3 h^{2}(\gamma+1)^{3}+h\left[3 \gamma^{4} z+\gamma^{3}(3 z \lambda+11 z+6)+\right.\right. \\
& \gamma^{2}(9 z \lambda+16 z+12)+\gamma(9 z \lambda+11 z+6)+3 z \lambda+ \\
& 3 z]+\gamma\left\{\gamma^{3}\left[3 z^{2} \lambda+z(z+4)\right]+\gamma^{2}[4 z(2 z+1) \lambda+\right. \\
& 2 z(z+6)+3]+\gamma[z(9 z+8) \lambda+2 z(z+6)+3]+ \\
& z[4(z+1) \lambda+z+4]\}\}\},
\end{aligned}
$$




$$
\begin{aligned}
A_{3}= & \gamma^{2}\left\{z^{2} \gamma^{2}\{\gamma[(z-1) \lambda-1]+(z-2) \lambda-1\}\right. \\
& -h\left\{3 h^{2}(\gamma+1)^{2}+h\left[3 \gamma^{3} z+\gamma^{2}(3 z \lambda+10 z+3)+\right.\right. \\
& \gamma(6 z \lambda+10 z+3)+3 z \lambda+3 z]+z \gamma\left\{\gamma^{2}[3 z \lambda+\right. \\
& 2(z+1)]+\gamma[(7 z+2) \lambda+3(z+2)]+(5 z+2) \lambda+ \\
& 2(z+1)\}\}\},
\end{aligned}
$$

$$
\begin{aligned}
A_{4}= & h \gamma^{3}\left\{h^{2}(\gamma+1)+h z\left[\gamma^{2}+\gamma(\lambda+3)+\lambda+1\right]+\right. \\
& \left.z^{2} \gamma[\gamma(\lambda+1)+2 \lambda+1]\right\}
\end{aligned}
$$

Cardan's formula yields the solution of eq. A.9), from which the dynamic range can be numerically obtained.
[1] A. L. Hodgkin and A. F. Huxley, J. Neurophysiol. 117, 500 (1952).

[2] S. S. Stevens, Psychophysics: Introduction to its Perceptual, Neural and Social Prospects (Wiley, New York, 1975).

[3] J.-P. Rospars, P. Lánský, P. Duchamp-Viret, and A. Duchamp, BioSystems 58, 133 (2000).

[4] J.-P. Rospars, P. Lánský, P. Duchamp-Viret, and A. Duchamp, Eur. J. Neurosci. 18, 1135 (2003).

[5] T. A. Cleland and C. Linster, Neural Computation 11, 1673 (1999).

[6] R. A. Normann and F. S. Werblin, J. Gen. Physiol. 63, 37 (1974).

[7] F. S. Werblin, J. Gen. Physiol. 63, 62 (1974).

[8] F. S. Werblin and D. R. Copenhagen, J. Gen. Physiol. 63, 88 (1974).

[9] K. J. Kim and F. Rieke, J. Neurosci. 23, 1506 (2003).

[10] A. Borst, V. L. Flanagin, and H. Sompolinsky, Proc. Natl. Acad. Sci. USA 102, 6172 (2005).

[11] M. R. Deans, B. Volgyi, D. A. Goodenough, S. A. Bloomfield, and D. L. Paul, Neuron 36, 703 (2002).

[12] L. S. Furtado and M. Copelli, Phys. Rev. E 73, 011907 (2006).

[13] M. Copelli, A. C. Roque, R. F. Oliveira, and O. Kinouchi, Phys. Rev. E 65, 060901 (2002).

[14] M. Copelli, R. F. Oliveira, A. C. Roque, and O. Kinouchi, Neurocomputing 65-66, 691 (2005).

[15] M. Copelli and O. Kinouchi, Physica A 349, 431 (2005).

[16] O. Kinouchi and M. Copelli, Nature Phys. 2, 348 (2006).

[17] A.-C. Wu, X.-J. Xu, and Y.-H. Wang, Phys. Rev. E 75, 032901 (2007).

[18] M. Copelli and P. R. A. Campos, Eur. Phys. J. B 56, 273 (2007).

[19] M. Brecht, M. Schneider, B. Sakmann, and T. W. Margrie, Nature 427, 704 (2004).
[20] S. Hestrin and M. Galarreta, Trends Neurosci. 28, 304 (2005).

[21] R. W. Friedrich and S. I. Korsching, Neuron 18, 737 (1997).

[22] M. Wachowiak and L. B. Cohen, Neuron 32, 723 (2001).

[23] J. Joo and J. L. Lebowitz, Phys. Rev. E 70, 036114 (2004).

[24] J. Marro and R. Dickman, Nonequilibrium Phase Transition in Lattice Models (Cambridge University Press, Cambridge, 1999).

[25] J. Adler and J. A. M. S. Duarte, Phys. Rev. B 35, 7046 (1987).

[26] J. Adler, J. Berger, J. A. M. S. Duarte, and Y. Meir, Phys. Rev. B 37, 7529 (1988).

[27] S. P. Obukhov, Physica A 101, 145 (1980).

[28] H. K. Janssen, Z. Phys. B 42, 151 (1981).

[29] H. K. Janssen, Z. Phys. B 58, 311 (1985).

[30] P. Grassberger, Z. Phys. B 47, 365 (1982).

[31] J. M. Greenberg and S. P. Hastings, SIAM J. Appl. Math. 34, 515 (1978).

[32] P. R. Roelfsema, A. K. Engel, P. König, and W. Singer, Nature 385, 157 (1997).

[33] G. B. Ermentrout and D. Kleinfeld, Neuron 29, 33 (2001).

[34] J. M. Beggs and D. Plenz, J. Neurosci. 23, 11167 (2003).

[35] J. M. Beggs and D. Plenz, J. Neurosci. 24, 5216 (2004).

[36] D. Plenz and T. C. Thiagarajan, Trends Neurosci. 30, 101 (2007).

[37] D. R. Chialvo, Physica A 340, 756 (2004).

[38] V. M. Eguíluz, D. R. Chialvo, G. A. Cecchi, M. Baliki, and A. V. Apkarian, Phys. Rev. Lett. 94, 018102 (2005).

[39] O. Sporns, D. R. Chialvo, M. Kaiser, and C. C. Hilgetag, Trends Cog. Sci. 8, 418 (2004). 